\title{
16
}

\section{How Interpretation of Indistinct Covert Recordings Can Lead to Wrongful Conviction: A Case Study and Recommendations for Reform}

\section{Helen Fraser $^{1}$}

\section{Introduction and Overview}

Covert recording ('bugging') is now authorised in almost every major police investigation. Unfortunately, because the need for secrecy compromises control over recording conditions, the audio is often indistinct. Legal practice regarding the use of indistinct covert recordings in trials has evolved haphazardly over the past 30 years, with no consultation of phonetic science. This has resulted in a number of anomalies, notably the fact that detectives are allowed (as 'ad hoc experts') to present their own transcripts of indistinct audio to 'assist' the jury in interpreting the audio evidence. 
This chapter ${ }^{2}$ highlights problems with this practice via a case study of a murder conviction obtained on the basis of a demonstrably inaccurate police transcript, then suggests directions for reform.

\section{What Are Covert Recordings?}

Covert recordings are conversations recorded secretly, without the knowledge of one or more of the speakers, via telephone intercept, hidden listening device or similar means. Legally obtained covert recordings can provide powerful evidence in criminal cases, capturing speakers giving information or making admissions they might not be willing to reveal openly in court.

While covert recordings are highly useful, they have a major drawback. Since it is difficult to control the recording conditions, the audio is frequently of extremely poor quality, to the extent it is impossible to make out what is said on one hearing. Several examples are given at forensictranscription. com.au, including an excerpt from a recording used in a murder trial that forms the basis of an extensive case study summarised briefly here.

This excerpt features a whispered conversation evidently about a pact related to the murder. The issue to be resolved by the trial was whether this was a pact to conceal the murder (making the speaker an accessory after the fact, a crime to which the defendant admitted) or a pact to commit the murder (making the speaker an accessory before the fact, which he denied).

This recording was the only direct evidence bearing on this issue, all the other evidence being described by the judge as circumstantial. The problem is that, on one or even several hearings, the audio is indecipherable. This is a common occurrence, that the law has had to deal with since covert recordings started to be used routinely by police in the 1980s.

2 This chapter summarises material from several previous publications. The following in particular contains extensive background references: H Fraser, 'Transcription of Indistinct Covert Recordings Used as Evidence in Criminal Trials' in H Selby and I Freckelton (eds), Expert Evidence (Thomson Reuters, 2015). 


\section{Standard Practice}

Following Butera (1987), ${ }^{3}$ a range of practices have been standardised in relation to indistinct covert recordings.

First, since it is clearly not convenient to play audio repeatedly in court, the judge can allow a transcript to assist the jury in making out what is said in the recording. Second, the transcript can be prepared by detectives working on the case. Many citizens are surprised or even alarmed to find that police transcripts are used in this way. However, when the reasons are explained, they are found to have a certain logic.

Indistinct audio is too hard for regular transcription agencies. However, since most covert recordings are obtained on behalf of police, detectives create their own transcripts as part of their investigations. When lawyers listen to an indistinct recording with the aid of a police transcript, it seems evident that the transcript is at least largely accurate. It appears that police transcribers, by listening many times to the indistinct audio, have been able to make out the words, and that their transcript is able to help listeners, saving them the trouble of repeated listening.

Of course it is clear that there are some risks in allowing police transcripts to assist in this way. In legal contexts, transcripts are often used as a convenient replacement for the audio itself. This makes some sense for reliable transcripts of overt recordings, such as court proceedings or police interviews, but it is essential that a jury should not be allowed simply to read a (possibly misleading) police transcript as a factual representation of audio used in evidence.

To avoid this, legal practice has developed a number of checks and balances. First, the defence is expected to scrutinise police transcripts thoroughly. In the event of disagreement over particular words or sections, the judge is expected to listen personally and make a ruling. Most commonly, this results in the decision being deemed 'a matter for the jury' - though it is open for the judge to exclude the police transcript, and there are precedents for this. 
In addition, the law insists the judge should carefully instruct the jury that the evidence is the audio, not the transcript. The transcript is provided only to assist them in hearing the recording, not as a replacement for it. After the audio has been played once in open court, it is given to the jury, who are encouraged to listen carefully in their own time, and form their own opinion as to what is said.

In essence, the transcript is provided, not as a factual representation of the recording, but as an opinion about its contents, for evaluation by the jury. In order to be allowed to offer an opinion (as opposed to factual evidence) in court, detectives provide transcripts in the role of so-called 'ad hoc expert'. ${ }^{4}$ An 'ad hoc expert' is someone who, though lacking genuine expertise in a particular discipline, is deemed to have 'specialised knowledge's in relation to a particular trial. With indistinct covert recordings, the specialised knowledge is understood to emerge from repeated listening to the audio.

\section{Spurious Reasoning}

This explanation usually eases the qualms of those who are initially uncomfortable with the concept of police transcripts being used to assist with the interpretation of indistinct audio. However, although its logic may seem plausible on the basis of common knowledge, from the point of view of phonetic science it is entirely spurious. Indeed the potential for injustice is far greater than recognised by those with general unease regarding use of police transcripts.

Phonetics (the science of speech) is a little known and much misunderstood subject, even among scientists from other disciplines. Many assume it simply adds technical detail to facts already widely known through general education. The reality is that the findings of phonetic science overturn many apparent truisms of everyday knowledge. In particular, ideas underpinning the legal practice just described, widely accepted as self-evidently true, turn out to be fallacies.

4 G Edmond and M San Roque, 'Quasi-Justice: Ad Hoc Expertise and Identification Evidence' (2009) 33 Criminal Law Journal 8.

5 Evidence Act 1995 (Cth) s 79. 


\section{A. Fallacy: Transcription is a mere 'secretarial skill'}

Transcripts are used frequently in legal contexts, where transcription is deemed a simple process of writing down what was said, the main requirement being an ability to spell. This view has limited validity for transcription of clear, overt recordings. However, in relation to covert recordings it is straightforwardly fallacious. Both determining what was said, and rendering it in an appropriate format, require complex, highlevel skills. As is well known in the language sciences, a transcript is never 'the' transcript. It can only ever be 'a' transcript. ${ }^{6}$

\section{B. Fallacy: The more you listen the more accurately you hear}

Listening many times is essential for creating a reliable transcript of indistinct audio. However, it is far from being sufficient. It is quite possible to listen many times and be wrong every time. Indeed, depending on the circumstances, listening repeatedly is more likely to entrench inaccurate perception than correct it.

\section{Fallacy: It is easy to pick up errors by checking the transcript against the audio}

When we listen to audio first without a transcript and then with a transcript, it seems that the transcript is aiding our perception. In fact it is creating our perception (as explained further below). Surprising as it may seem, the easiest way to change listeners' perception of indistinct audio is not to tamper with the audio itself, but simply to provide a different transcript. It is impossible to convey this effect adequately on paper (see examples at forensictranscription.com.au) but what it means is that errors are unlikely to be detected through normal 'checking'.

6 H Fraser, 'Transcription of Indistinct Forensic Recordings: Problems and Solutions from the Perspective of Phonetic Science' (2014) 1 Language and Law/Linguagem e Direito 5, ler.letras.up.pt/ site/default.aspx?qry=id05id1444id2692\&sum=sim. 


\section{The Fascinating Role of Expectation in Speech Perception}

Demonstrations of these fallacies, though well known in phonetic science, are generally found 'amazing' and 'fascinating' by those who first encounter them. 'Common knowledge' asserts that hearing is a straightforward process of picking up 'what is there to be heard'. However, the reality is that listeners' unconscious expectations play an enormous role in shaping speech perception. This is similar to the general phenomenon of cognitive bias, or suggestibility, ${ }^{7}$ but plays out rather differently with speech than with other kinds of perception.

In fact, the ability of expectation to mislead speech perception is not unknown in everyday life. It forms the basis of entertainment based on misheard song lyrics and other kinds of humour. However, it has a darker side in criminal law. In particular, it means all the checks and balances intended to ensure that a police transcript assists, rather than misleads, the jury are ineffective.

The 'pact' case study discussed earlier makes a good example. Experimental studies ${ }^{8}$ show that listeners who are given the excerpt 'cold' never hear anything about a pact. Only when they are led by context to understand that the conversation relates to some kind of pact, do they hear the word 'pact', and even then no one hears the exact words of the police transcript ('at the start we made a pact'). However, once the police transcript is shown, the interpretation 'at the start we made a pact' is readily accepted by a majority of listeners - who are then willing to offer an opinion as to whether the covert recording suggests the pact was to commit or conceal murder.

This could be interpreted, and indeed is often experienced by those going through the perceptual stages just described, as the transcript 'assisting' perception. This interpretation, however, is contradicted by the fact that phonetic analysis demonstrates 'at the start we made a pact' is an inaccurate transcription. Unfortunately, it is not so easy to state with

7 A M Ridley, F Gabbert and D J La Rooy, Suggestibility in Legal Contexts (Wiley-Blackwell, 2013) 254.

8 H Fraser and B Stevenson, 'The Power and Persistence of Contextual Priming: More Risks in Using Police Transcripts to Aid Jurors' Perception of Poor Quality Covert Recordings' (2014) 18 International Journal of Evidence and Proof 205. 
certainty exactly what was said, but it is something with the rhythmic structure of 'it's fucking payback' - quite different from that of 'at the start we made a pact'.

This demonstrates how expectations created by viewing a transcript can influence perception even when the transcript is manifestly wrong. The experience of being assisted by a reliable transcript is indistinguishable from the experience of being misled by an unreliable one.

As discussed above, this is a major problem for the standard legal concept that inaccuracies in a police transcript will be detected by ensuring it is checked against the audio by the defence, the judge, and the jury. A further finding of phonetic science creates even bigger problems for that concept. When participants are told that expert analysis reveals a transcript to be inaccurate, many still prefer the version they now seem to 'hear with their own ears', forgetting that it was only after being 'assisted' by the transcript that 'their own ears' heard anything remotely like the words it contains.

In considering the relevance of findings like these, it is worth recalling that juries evaluate police transcripts under conditions far less favourable for detection of inaccuracy than do participants in these experiments. Among other factors, participants hear the audio in short, carefully prepared sections, which they play repeatedly under a range of conditions deliberately constructed to reduce the influence of the transcript. Juries have neither the means nor the time to experience this.

Most importantly, participants first hear the audio 'cold', while a jury knows the content of the transcript in advance. This means a jury never has the experience of being 'unable to hear a word' before the transcript is presented, an important (though not always effective) factor in raising awareness that the transcript has influenced perception.

\section{A Flawed Process}

All this means the likelihood of misleading errors or omissions in police transcripts being detected is very low, as first prosecutors, then defence, then the judge, and ultimately the jury, are deeply but unwittingly influenced by what they see on the page. 
It also means the likelihood of police transcripts containing misleading errors or omissions is very high. What helps police hear indistinct audio that is opaque to others is not their repeated listening. It is their knowledge and assumptions about the context of the recording. To the extent such knowledge and assumptions are valid, they can indeed give valuable insight into the content of an indistinct recording. However, they are a double edged sword, which can mislead as easily as help. ${ }^{9}$

The potential for injustice is evident, and I am personally aware of an alarming number of cases where demonstrably inaccurate and misleading transcripts have been admitted as 'assistance' to the court. The case study described earlier recounts one of the most troubling.

In this trial, the defendant was found guilty of murder and sentenced to 30 years in prison, largely on the basis that a covert recording revealed him saying 'at the start we made a pact'. However, as discussed above, phonetic analysis makes clear that these words were not actually spoken.

In considering the import of this finding, it is useful to know that the murder was actually committed by this man's son (who confessed and was convicted and sentenced in a separate trial), at a time when the father had a solid alibi. The whole idea that the father was involved in any way arose through a detective erroneously believing he heard words which he interpreted as an admission that the father had masterminded the murder. Of course, once this idea had arisen, a good deal of 'circumstantial' evidence was able to be accumulated to support it. However, it is an open question how much might or might not have been made of that evidence without the (inaccurate) transcript to filter the interpretation of, first, investigators, and finally the jury. Recent demonstration in a very thorough, large-scale study ${ }^{10}$ of the role of 'tunnel vision' as a factor increasing the risk of wrongful conviction is surely relevant.

Unfortunately, expert analysis of the audio in this case occurred only after the defence had exhausted all avenues of appeal (none related to the transcript). The only recourse was to seek to have the case reopened via an application to review the conviction. This application has recently been

9 H Fraser, 'Issues in Transcription: Factors Affecting the Reliability of Transcripts as Evidence in Legal Cases' (2003) 10 International Journal of Speech Language and the Law 203.

10 J B Gould et al, Predicting Erroneous Convictions: A Social Science Approach to Miscarriages of Justice (National Institute of Justice, 2012). 
rejected in a ruling that disappointingly upholds fallacious ideas about speech and speech perception such as those discussed above (see case study at forensictranscription.com.au).

\section{The Problem and the Solution}

The law's aim of maximising the assistance juries can derive from police insights about the content of covert recordings, while minimising the effect of transcript inaccuracies, is entirely reasonable, and one that phonetic science can assist with. Unfortunately, the practices the law has so far developed to achieve this aim are ineffective. Essentially they bring back 'verballing' ${ }^{11}$ on a grand scale, without requiring conscious intention on the part of police, ${ }^{12}$ creating substantial problems of actual and potential injustice.

The most important step in solving any problem is to a gain good understanding of its causes. Otherwise attempted solutions simply create new problems.

Here, it is clear that the problems are caused by the law taking phonetic science into its own hands, unaware of how many ideas about speech and speech perception, still accepted as 'common knowledge', have been shown to be false. Equally clearly, then, a good solution is unlikely to be found by continuing the process of developing practice for handling speech evidence by creating and applying legal precedents. Even if the original judgment is valid, its application in subsequent cases may not be, as seen in the less-than-optimal response in the UK to a useful judgment ${ }^{13}$ criticising 'ad hoc expert' speaker identification evidence.

\footnotetext{
11 'Verballing' is claiming someone said something when in fact they didn't, notably in fabricated confessions. Evidence of verballing being used to obtain convictions was an important element in findings of police corruption in the 1990s (e.g. Wood, J R T (1997), Royal Commission into the New South Wales Police Service, Volume 1: Corruption (Sydney: Government of NSW).

12 H Fraser, 'Covert Recordings as Evidence in Court: The Return of Police "Verballing"?' [2013] The Conversation, theconversation.com/covert-recordings-as-evidence-in-court-the-return-of-policeverballing-14072.

13 See Rv Flynn [2008] 2 Cr App R 266.
} 
What is needed is wholesale reform ${ }^{14}$ of legal practice around the handling and admission of indistinct covert recordings. To quickly forestall a common kneejerk excuse for avoiding the issue, such a system would certainly not involve having every covert recording transcribed by an expert in phonetic science.

Rather it would involve designing a system that ensures transcripts are reliable before covert recordings enter the legal process. Deciding the exact nature of such a system requires discussion and research conducted in close collaboration between phonetic science and the law. However, once the will is in place, it need not be overly difficult. An excellent precedent is seen in the highly practical and universally appreciated system for creating and using recorded police interviews, implemented to prevent police 'verballing'.

14 Perhaps more accurately undoing of the unfortunate effects of reforms introduced by Butera. 
This text is taken from New Directions for Law in Australia: Essays in Contemporary Law Reform, edited by Ron Levy, Molly O’Brien, Simon Rice, Pauline Ridge and Margaret Thornton, published 2017 by ANU Press, The Australian National University, Canberra, Australia. 